\title{
MODELAGEM DA PREVISÃO DE VENDAS DE UMA UNIDADE AGRÍCOLA PRODUTORA DE BANANAS EM MISSÃO VELHA (CE)
}

\section{BANANA SALES FORECASTING MODEL OF AN AGRICULTURAL UNIT, IN CEARÁ, BRAZIL}

\author{
Jaqueline de Jesus Lima*E-mail: jaquelinedejesus@aluno.fapce.edu.br \\ Mauro Macedo de Oliveira* E-mail: mauro.oliveira@fapce.edu.br \\ ${ }^{*}$ Centro Universitário Paraíso (UNIFAP), Juazeiro do Norte, Ceará
}

\begin{abstract}
Resumo: A importância de planejar as necessidades futuras surge das exigências dos processos decisórios, uma vez que todos os negócios estão suscetíveis a imprevistos e necessitam ter flexibilidade. No processo de realização do planejamento estratégico é de grande importância incluir a previsão de demanda para dimensionar a capacidade produtiva. Um estudo de previsão demanda foi desenvolvido numa empresa de 330 hectares que cultiva bananas prata e nanica, localizada na cidade de Missão Velha, estado do Ceará. Este trabalho consiste numa pesquisa explicativa com abordagem quantitativa, onde inicialmente foram levantados os dados históricos de vendas e em seguida verificados seus comportamentos a partir de testes estatísticos. Foi escolhida a banana prata pela sua representatividade para a empresa. Identificou-se comportamentos de tendência e sazonalidade. Após essa constatação aplicou-se os métodos ARIMA e Holt-Winters a fim de identificar o método que melhor se adequava, com o intuito de evitar desequilíbrios entre a equipe de produção e comercial. O método que apresentou os melhores resultados foi o ARIMA $(1,1,1)$, tendo como critério para a validação o MAD, MAPE e MSD.
\end{abstract}

Palavras-chave: Previsão de demanda. Planejamento e controle da produção. Bananicultura.

Abstract: The importance of planning future needs arises from the requirements of decision-making processes, since all businesses are susceptible to unforeseen events and need to be flexible. In the process of carrying out aggregate planning, it is important to know the demand to scale the productive capacity. A forecast study was carried out at a company in Ceará, Brazil, where burro and yellow cavendish bananas are produced on 330 acres. This work is an explanatory and quantitative research, in which historical sales data were initially collected and then their behavior was verified using statistical tests. The burro banana was chosen for its representativeness to the company. Growth trend and seasonality behaviors were identified. After this verification, the ARIMA and Holt-Winters methods were applied for the burro banana in order to identify the method that was best suited. This becomes essential to avoid imbalances between the production and commercial team. The method that presented the best results were ARIMA $(1,1,1)$ with MAD, MAPE and MSD as validation criteria.

Keywords: Demand forecast. Planning and production control. Banana production.

\section{INTRODUÇÃO}

A necessidade de planejar as demandas futuras surge das exigências dos processos decisórios na obtenção de recursos, e a questão está exatamente entre o momento da tomada da decisão e o momento em que o efeito da decisão tomada começará a ser percebida, então quanto antes a decisão for tomada maior será o tempo disponível para a preparação do que irá acontecer (CORRÊA; GIANESI; 
CAON, 2006). A importância da previsão de demanda pode ser percebida quando Tubino (2009, p.15) comenta: "A previsão da demanda é a base para o planejamento estratégico da produção, vendas e finanças de qualquer empresa".

A previsão não é apenas antecipar o futuro com informações aleatórias ou através de adivinhas, ela está ligada a um conjunto de métodos e a experiência de mercado daqueles que realizarão as previsões, que ao final, um conjunto de dados representará a organização (FERNANDES; GODINHO FILHO, 2016). A previsão possibilita a adequação no uso das máquinas, a compra da quantidade necessária dos materiais no tempo correto e na programação das atividades dentro dos prazos adequados. Por representar grande importância para o planejamento das organizações é preciso ter bastante atenção pois as previsões não são exatas, elas apresentam erros, daí faz-se necessário ter cuidado e critério no momento da coleta de dados e na escolha do modelo para que os erros sejam mínimos (MARTINS; LAUGENI, 2015).

Diante da importância de planejar as demandas futuras e prever as mais diversas situações é que identificamos que essa necessidade se aplica a todos os ramos da atividade econômica, onde destacaremos aqui o setor agrícola brasileiro que possui bastante relevância na economia mundial. Isso pode ser visto nos dados da Organização das Nações Unidas para Alimentação e Agricultura - FAO que registrou no ano de 2017 uma produção de banana de 6.675 .100 toneladas no Brasil, colocando o mesmo em $4^{\circ}$ lugar na produção mundial. No Nordeste foram 2.251.907 toneladas representando uma participação de 33,74\% do país. Dessa produção o estado do Ceará contribuiu com 393.738 toneladas e Missão Velha com 42.982 toneladas conforme dos dados Instituto de Geografia e Estatística - IBGE (2017).

Esse estudo foi desenvolvido numa empresa que atua no cultivo de 330 hectares de produção de banana em Missão Velha-Ceará, cultivando as variedades prata e nanica. A banana prata é responsável pela maior parte da área cultivada $(93,43 \%)$ bem como pelo maior faturamento $(85,00 \%)$ da empresa.

Fundada em 1996, foi a pioneira em bananicultura na região do Cariri cearense. A empresa participa de toda a cadeia produtiva, desde a produção até a distribuição nos supermercados. Atualmente a única ação que a empresa realiza relacionada à demanda consiste na verificação da quantidade de cachos disponíveis para comercialização 18 semanas antes de serem colhidas para a de banana prata, 
podendo se estender até 21 semanas. Para banana nanica a antecedência é de 12 semanas podendo se estender até 14 semanas. Essas variações se dão por conta das mudanças climáticas. Assim a quantidade de cachos é inserida em um sistema informatizado que fica restrita apenas ao conhecimento do setor da produção. Já o departamento comercial efetua, no final de cada semana, os pedidos que serão atendidos na semana posterior. Quando ocorrem situações onde as vendas são inferiores ou superioras à quantidade disponível em campo, a tomada de decisão para solucionar esses problemas é realizada de maneira repentina, causando desarmonia entre a produção e o setor comercial.

Portanto esse estudo tem como objetivo realizar uma previsão de vendas para a banana do tipo prata, baseada na série histórica de vendas da empresa, com intuito de evitar desequilíbrios entre a equipe de produção e departamento comercial, proporcionando melhor sinergia entre esses setores e um melhor planejamento estratégico da produção.

\section{FUNDAMENTAÇÃO TEÓRICA}

\subsection{Bananicultura}

A origem da bananeira é atribuída ao sudeste asiático onde foram identificados indícios do cultivo por volta de 8000 a.C. Nas ilhas atlânticas, Brasil e costa ocidental africana, os colonizadores portugueses iniciaram o cultivo de forma mais estruturada nos séculos XV e XVI. É uma fruta cujo sabor agrada muitos paladares e pode ser consumida com muita facilidade. A banana é fonte de energia, vitaminas e minerais e possui um custo acessível aos mais diversos públicos (SILVA et al., 2016). Além de ser uma fruta que complementa a dieta da população, para muitos países, a banana tem grande importância social e econômica, atuando como fonte de renda para várias famílias de agricultores, proporcionando oportunidades de empregos no campo e na cidade e colaborando para o desenvolvimento das regiões envolvidas em sua produção (FIORAVANÇO, 2003).

A bananeira é uma planta bem exigente quanto aos nutrientes, também é muito sensível às questões físicas do solo como: ar, água, temperatura e a resistência dela quanto ao desenvolvimento das raízes. Solos que não apresentam condições adequadas ao cultivo podem resultar em baixa produtividade (SOUZA; 
BORGES; SILVA, 2016). Por esse motivo as questões exigidas para o gerenciamento do cultivo não estão relacionadas somente aos aspectos produtivos em si, mas também em tudo que interfere no meio onde a planta está sendo cultivada.

As regiões norte e nordeste apresentam algumas vantagens em relação as outras regiões, como uma baixa incidência de doenças, oferta de fruta regular e qualidade satisfatória. Isso é possível por conta das condições climáticas dessas regiões e pelo manejo adequado de irrigação (ALMEIDA; SOUZA; CORDEIRO, 2000).

\subsection{Planejamento e Controle de Produção (PCP)}

O planejamento e controle estão relacionados na combinação das demandas dos clientes com a capacidade dos recursos produtivos disponíveis para entregar o que foi solicitado. Não é apenas estabelecer estratégias de vendas e vender, é preciso inicialmente reconhecer se a capacidade instalada agrega condições para atender a demanda, e se essa estrutura garante o máximo de rentabilidade. Portanto a capacidade instalada deve estar em consonância com o que se planeja comercializar (SLACK; CHAMBERS; JOHNSTON, 2015).

As atividades do PCP são exercidas em nível estratégico, a longo prazo, na construção do planejamento estratégico de produção; em nível tático, no médio prazo, no planejamento-mestre de produção; e no curto prazo, em nível operacional, na preparação da programação da produção (TUBINO, 2017).

Quando se comparam processos produtivos, nota-se que os riscos que os empresários rurais estão expostos são mais significativos em relação à atividade industrial. Eles estão sujeitos às situações que podem ou não ser controladas, tais como: chuvas, secas, solo, umidade etc. Nesse contexto os conceitos de PCP são aplicados ao agronegócio inicialmente pelo desejo de se reduzir os custos (PAPALARDO; MACHADO; SACOMANO, 2018). Isso nos leva a entender que além das intempéries que precisam ser bem gerenciadas, é primordial uma correta utilização dos recursos disponíveis para garantir a rentabilidade do cultivo. 


\subsection{Previsão de Demanda}

Estudar a situação atual do mercado e suas tendências, assim como analisar dados históricos da empresa e sua capacidade instalada são informações de grande importância no auxílio do processo de planejamento e previsão das demandas futuras.

Para que seja possível realizar uma boa previsão é muito importante que a empresa saiba fazer uso das ferramentas necessárias para tal. Nesse sentido destaca-se a montagem de uma base de dados com históricos de vendas que exponham as mudanças e comportamentos que ocorreram no passado, bem como a utilização de modelos matemáticos adequados que auxiliarão na explicação do comportamento da demanda futura (CORRÊA; GIANESI; CAON, 2007). Um modelo depende principalmente do comportamento da série que se está analisando. Uma série temporal pode apresentar até quatro particularidades distintas em seu comportamento: média, sazonalidade, ciclo e tendência (MAKRIDAKIS; WHEELWRIGHT; HYNDMAN, 1998).

Identifica-se média quando os valores da série variam em torno de uma média constante. Comportamento sazonal quando possuir padrões cíclicos de variação e que em intervalos relativamente constantes de tempo se repetem. $O$ comportamento cíclico se faz presente quando a série apresenta variações ascendentes e descendentes, no entanto, os intervalos de tempo não são regulares. E a série apresentará tendência quando em um longo período apresentar um comportamento de crescimento ou declínio (PELLEGRINI, 2000).

Saber que a demanda por seus produtos está aumentando ou diminuindo não é o suficiente, conhecer o quanto está mudando é de grande importância para o processo de planejamento da empresa. E quando se tem conhecimento do que está mudando é possível traçar ações para melhor conduzir essas mudanças (SLACK; CHAMBERS; JOHNSTON, 2015).

Para se construir um modelo de previsão de demanda deve-se inicialmente definir seus objetivos, onde serão escolhidos os produtos e os detalhes esperados da previsão. Em seguida realiza-se a coleta e análise dos dados, levando em consideração que quanto mais dados coletados mais confiáveis serão os resultados. A partir do comportamento dos dados, seleciona-se a técnica mais adequada aplicando-a de forma a se obter projeções. Finaliza-se com o monitoramento do 
modelo a partir dos erros entre a demanda real e a prevista a fim de se verificar a acuracidade do modelo utilizado (TUBINO, 2017).

\subsubsection{Técnicas de previsão}

Dentre as técnicas de previsão existem as qualitativas e quantitativas. As técnicas qualitativas estão relacionadas com dados subjetivos que são mais difíceis de ser representados em números. Ela está mais voltada para a experiência de pessoas estratégicas que conhecem o produto e o mercado. Já as técnicas quantitativas abordam uma análise numérica de dados passados, e nesse caso, elas podem ser subdivididas em séries temporais e técnicas baseadas em correlações (TUBINO, 2017).

As séries temporais estão relacionadas a um conjunto de dados que estão ordenados no tempo. As previsões que utilizam essa abordagem se baseiam na ideia de que será possível ter uma previsão levando em consideração que questões que influenciaram a demanda no passado continuarão atuando no futuro. Assim, é necessário inicialmente que seja compreendido como se comportaram os dados no decorrer do tempo para que se possa determinar o modelo mais adequado a ser utilizado (FERNANDES; GODINHO FILHO, 2016).

Segundo Krajewski, Ritzman, Malhotra (2009) os métodos utilizados para as séries temporais têm como base dados históricos da variável dependente. Desse modo esses métodos estão embasados na hipótese de que o padrão anterior continuará ocorrendo no futuro.

Os métodos matemáticos buscam compreender como os dados se comportaram e projetar o futuro a partir do que foi compreendido sobre os dados em questão. As séries temporais são os métodos mais simples e mais utilizados para realizar previsões. Uma boa elaboração destes resultará na obtenção de resultados bastante satisfatórios (TUBINO, 2009).

Considerando as séries temporais, abordaremos a seguir os principais métodos que são utilizados para realização de previsões de demanda.

\subsubsection{Média Móvel}

O modelo de média móvel se adequa quando os dados não apresentam 
tendência de aumentar ou diminuir de maneira acentuada, ou seja, há um comportamento com poucas variações que se aproxima da média (CORRÊA; GIANESI; CAON, 2007).

A equação deste modelo é apresentada na Equação 1, onde $D_{t}$ é a demanda real do período $t, n$ é o número total de períodos da média e $\mathrm{F}_{\mathrm{t}+1}$ que é a previsão para o período (KRAJEWSKI; RITZMAN; MALHOTRA, 2009).

$\mathrm{F}_{\mathrm{t}+1}=\frac{\text { Soma das últimas } n \text { demandas }}{n}=\frac{\mathrm{D}_{\mathrm{t}}+\mathrm{D}_{\mathrm{t}-1}+\mathrm{D}_{\mathrm{t}-2}+\cdots \mathrm{D}_{\mathrm{t}-\mathrm{n}+1}}{n}$

\subsubsection{Média Móvel Ponderada}

Para este método pode ser atribuído a cada dado histórico da média um peso específico, levando em consideração que a soma de todos os pesos deverá ser igual a 1. O cálculo para essa média é realizado através da multiplicação de cada peso pelo valor do período, finalizando com a soma de todos os resultados. Este método tem condições de ser aplicado a períodos sazonais atribuindo a eles um peso maior. $\mathrm{Na}$ Equação 2 tem-se um exemplo onde foi aplicado o peso 0,50 para o período mais recente, ao segundo 0,30 e o terceiro 0,20 (LÉLIS, 2012).

$F_{t+1}=0,50 D_{t}+0,30 D_{t-1}+0,20 D_{t-2}$

\subsubsection{Método da Suavização Exponencial Simples}

O método da suavização exponencial simples é indicado quando a demanda não possui tendência ou sazonalidade (CHOPRA; MEINDL, 2003).

$\mathrm{Na}$ suavização exponencial simples a previsão para período será a previsão do anterior corrigida pelo erro ocorrido no período anterior, a esse erro será atribuído um peso $\alpha$ (FERNANDES; GODINHO FILHO, 2016).

$S_{T}=S_{T-1}+\alpha\left(D_{T-1}-S_{T-1}\right)$ 


\subsubsection{Modelo de Holt}

Quando a demanda estudada possui tendência de crescimento ou declínio o método da suavização exponencial acaba não se adequando bem, pois pode gerar previsões maiores ou menores que a realidade. Diante disso, para corrigir essa tendência temos a suavização exponencial com tendência ou método de Holt, que utilizará para a previsão duas constantes de suavização. Assim além da constante de suavização alfa $(\alpha)$ será utilizado a constante de suavização beta $(\beta)$, que será responsável por reduzir o impacto do erro entre o valor real e o previsto (CHASE; JACOBS; AQUILANO, 2006).

$$
\begin{aligned}
& L_{t}=\alpha \cdot Y_{t}+(1-\alpha) \cdot\left(L_{T}+b_{t-1}\right) \\
& b_{t}=\beta \cdot\left(L_{t}+L_{t-1}\right)+(1-\beta) \cdot\left(b_{t-1}\right) \\
& F_{t+m}=L_{t}+m \cdot b_{t}
\end{aligned}
$$

\subsubsection{Modelo de Holt-Winters}

Esse modelo é adequado para demandas em que são identificadas a ocorrência de tendência linear e sazonalidade. O modelo pode ser aditivo, onde no decorrer do tempo a variação da amplitude sazonal é constante, ou multiplicativo em que a amplitude da variação sazonal poderá diminuir ou aumentar em função do tempo (PELLEGRINI; FOGLIATTO, 2001).

Para a construção do modelo são necessários três parâmetros - a realiza o ajuste da estimativa de nível à série amortecida, $\beta$ é a taxa de inclinação e $\delta$ o fator sazonalidade. O algoritmo do modelo é representado pelas Equações 7,8,9 e 10.

$$
\begin{aligned}
& \tilde{X}_{t}=(1-\beta)\left(\frac{x_{t}}{F_{t-s}}\right)+\beta\left(\tilde{X}_{t-1}+R_{t-1}\right. \\
& R_{t}=(1-\alpha) \cdot\left(X_{t}-\tilde{X}_{t-1}\right)+\alpha \cdot R_{t-1} \\
& F_{t}=(1-\delta) \cdot\left(\frac{X_{t}}{\hat{x}_{t}}\right)+\delta F_{t-s} \\
& \hat{X}_{t}(h)=\left(\tilde{X}_{t}+h \times R_{t}\right) \times F_{t-s+h}
\end{aligned}
$$


A Equação 7 é responsável pelo reajuste das estimativas de nível, a Equação 8 se encarrega do reajuste da taxa de crescimento, ou seja, tendência e a 9 realiza os ajustes sazonais. $O$ algoritmo é encerrado com a equação 10 que realiza a previsão (ROSSI; NEVES, 2014).

\subsubsection{Modelo Autorregressivo (AR)}

No modelo autorregressivo o valor atual $\left(\mathrm{x}_{\mathrm{t}}\right)$ da série temporal é explicado como uma função de $\mathrm{p}$ valores anteriores, $\mathrm{x}_{\mathrm{t}-1} ; \mathrm{x}_{\mathrm{t}-2} ; \ldots ; \mathrm{x}_{\mathrm{t}-\mathrm{p}}$, no qual $\mathrm{p}$ define o número de valores do passado necessário para que seja possível prever o valor atual (SHUMWAY; STOFFER, 2011). O modelo $A R(p)$ pode ser expresso da seguinte forma:

$x_{t}=\phi_{1} x_{t-1}+\phi_{2} x_{t-2}+\cdots+\phi_{p} x_{t-p}+\omega_{t}$

\subsubsection{Modelo de Médias Móveis (MA)}

O processo de $M A(q)$ resume-se a uma associação linear de termos de erro de ruído branco, onde temos $Y$ no intervalo t que é representado por uma constante adicionado de uma média móvel dos termos de erros atuais e passados (GUAJARATI; PORTER, 2011). O modelo MA(q) é apresenta na Equação 12.

$Y_{t}=\mu+\beta_{0} u_{t}+\beta_{1} u_{t-1}+\beta_{2} u_{t-2}+\cdots+\beta_{q} u_{t-q}$

\subsubsection{Modelo Autorregressivo de Médias Móveis (ARMA)}

Fazendo a junção do método autorregressivo (AR) com o de médias móveis (MA) chegamos ao modelo ARMA (p,q). Esse modelo é adequado quando se deseja um número não muito grande de parâmetros (BOX et al., 2016; MORETTIN; TOLOI, 1981) que pode ser representado conforme a equação (13).

$\tilde{z}_{t}=\phi_{1} \tilde{z}_{t-1}+\cdots+\phi_{p} \tilde{z}_{t-p}+a_{t}-\theta_{1} a_{t-1}-\cdots-\theta_{q} a_{t-q}$ 
Assim tem-se como operadores autoregressivos e de médias móveis $\phi(B)$ e $\theta(\mathrm{B})$, respectivamente. Com isso podemos reescrever esse modelo de maneira compacta conforme Equação 14 (MORETTIN; TOLOI, 1981).

$\phi(B) \tilde{Z}_{t}=\theta(B) a_{t}$

\subsubsection{Modelo Autorregressivo Integrado de Médias Móveis (ARIMA)}

O método ARIMA é um modelo que busca assimilar na série o comportamento da correlação ou da autocorrelação dos valores e assim realizar a previsão. Com a combinação do componente autorregressivo (AR), do filtro de integração (I) e das médias móveis (MA) tem-se como resultado esse modelo (KIRCHNER, 2006).

Para a aplicação do método ARIMA é necessário uma série temporal estacionária ou uma série que passe a ser estacionária depois de passar pelo processo de diferenciação (GUAJARATI; PORTER, 2011).

Os modelos AR, MA e ARMA são indicados para séries estacionárias, ou seja, aquelas que se manifestam no tempo ao redor de uma média uniforme. Por isso algumas séries precisam ser diferenciadas, como mostra a Equação 15, para se tornar estacionária (MORETTIN; TOLOI, 2006).

$W_{t}=Z_{t}-Z_{t-1}=(1-B) Z_{t}=\Delta Z_{t}$

Assim temos a Equação 16, representando o modelo ARIMA (p, d, q), onde as ordens $p$ e q são respectivamente $\phi(B)$ e $\theta(B)$ e inclui-se também a diferenciação d (MORETTIN; TOLOI, 2006).

$\phi(B) \Delta^{d} Z_{t}=\theta(B) a_{t}$

\subsubsection{Avaliação do método}

Após tomar conhecimento do comportamento dos dados é preciso escolher o melhor a ser aplicado. Desta forma o método ideal será aquele que melhor representar a variação da demanda no histórico de dados. Assim, quanto menor o erro entre o valor real e o valor previsto, mais próximo da demanda real o método estará (GONÇALVES, 2013). 
O modelo utilizado deve ser constantemente monitorado para mensuração dos erros de previsões. Os erros podem ocorrer por variação aleatória no mercado ou por um eventual equívoco na escolha do método de previsão. Podemos definir o

erro de um período como a diferença entre a demanda real e a previsão no período (FERNANDES; GODINHO FILHO, 2016), como definido na equação (17).

$\mathrm{e}_{t}=d_{t}-P_{t}$

Algumas medidas para controle do erro são apresentadas a seguir:

\subsubsection{Desvio Absoluto Médio (MAD)}

O MAD é muito utilizado pois é facilmente compreendido. Ele é resultado da média dos erros de previsão de uma série de períodos sem levar em consideração se o erro foi a cima ou a baixo do valor previsto, ou seja, considera o valor absoluto (KRAJEWSKI; RITZMAN; MALHOTRA, 2009). A fórmula do MAD é mostrada na Equação 18.

$\operatorname{MAD}=\frac{\Sigma\left|E_{t}\right|}{n} \hat{y}$

\subsubsection{Erro Percentual Absoluto Médio (MAPE)}

O MAPE é utilizado para fazer a relação entre o erro de previsão com a demanda, sendo proveitoso para posicionar a previsão de um ponto de vista mais adequado (KRAJEWSKI; RITZMAN; MALHOTRA, 2009). O resultado do mesmo é apresentado em percentual e pode ser calculado conforme a Equação (19).

MAPE $=\frac{\left(\Sigma\left|E_{t}\right| / D_{t}\right)(100)}{n}$ 


\subsubsection{Desvio Padrão Quadrático da Média (MSD)}

O MSD é bem comum quando se trata de ajuste de dados de séries temporais. Tem-se $y_{t}$ como sendo os valores dos dados da série, $\hat{y}_{t}$ são os ajustes que foram feitos e $\mathrm{n}$ é o número de períodos que estão sendo analisados. Assim quanto menor o MSD melhor será o ajuste do método (ACTION, 2019). A Equação (20) mostra como é realizado o cálculo do MSD.

$$
M S D=\frac{\sum_{t=1}^{n}\left|\left(y_{t}-\hat{y}_{t}\right)\right|^{2}}{n}
$$

\section{METODOLOGIA DA PESQUISA}

Trata-se de um estudo de caso que foi desenvolvido na empresa Sitio Barreiras Fruticultura através de uma pesquisa explicativa com abordagem quantitativa. Inicialmente fez-se o levantamento dos dados históricos de vendas com base em arquivos disponíveis no sistema informatizado da empresa. Esses dados foram tabulados, em seguida identificou-se o comportamento da série por meio de análises estatísticas, aplicou-se métodos de previsão conforme indicação da literatura, e por fim comparou-se a eficiência dos mesmos e identificou-se o mais adequado.

O histórico de vendas das bananas do tipo prata obtido abrangeu desde o mês de janeiro de 2009 até setembro de 2019, totalizando 129 períodos. A empresa costuma registrar suas informações em semanas, sendo assim para os dados coletados foi utilizado como regra de fechamento do mês o seu último sábado.

Para compreender o comportamento da série temporal foram realizados os testes de Kruskal-Wallis e Friedman para identificar a existência de sazonalidade, e os testes Wald-Wolfwitz, Cox-Stuart e Mann-Kendall para perceber algum comportamento de tendência. Para a realização dos testes utilizou-se a aplicação Action Stat (versão gratuita), uma extensão instalada no Microsoft Excel, sendo definido como grau de significância o valor 0,05 . Assim quando o $p$-valor foi menor que o grau de significância rejeitou-se a hipótese nula e aceitou-se a hipótese alternativa, quando maior fez-se o contrário.

Após realização desses testes estatísticos, realizou-se a previsão para 6 
(seis) meses ainda utilizando o Action Stat. Aplicou-se o método ARIMA com a opção "automático" assinalada para que o próprio algoritmo pudesse gerar os parâmetros $(p, d, q)$ que melhor se adequassem aos dados fornecidos. Também se aplicou o método de Holt-Winters, pois este é um método que responde bem às séries que possuem tendência e sazonalidade. Com os resultados das previsões avaliou-se a acurácia de cada método pelo MAD (desvio médio absoluto), MAPE (erro absoluto médio percentual) e MSD (Desvio padrão quadrático da média).

\section{RESULTADOS E DISCUSSÃO}

A série histórica das vendas de banana prata, tabulada em 129 períodos compreendendo os meses de janeiro de 2009 a setembro de 2019, é mostrada no Anexo 1. Para melhor visualização do comportamento da série temporal, foi gerado uma curva apresentada na Figuras 1. Apenas pela curva já foi possível inferir algumas hipóteses sobre o comportamento da série, porém isso não é o suficiente para se obter uma análise mais concreta e confiável.

Os testes de Kruskal-Wallis e Friedman utilizados na identificação da sazonalidade resultaram em p-valor de 0,001761924 e 0,007478962 respectivamente. Indicando que deve ser rejeitado a hipótese nula que não há sazonalidade e aceitar a hipótese alternativa de que há sazonalidade determinística com uma frequência de 12 meses.

Figura 1 - Série temporal da banana prata

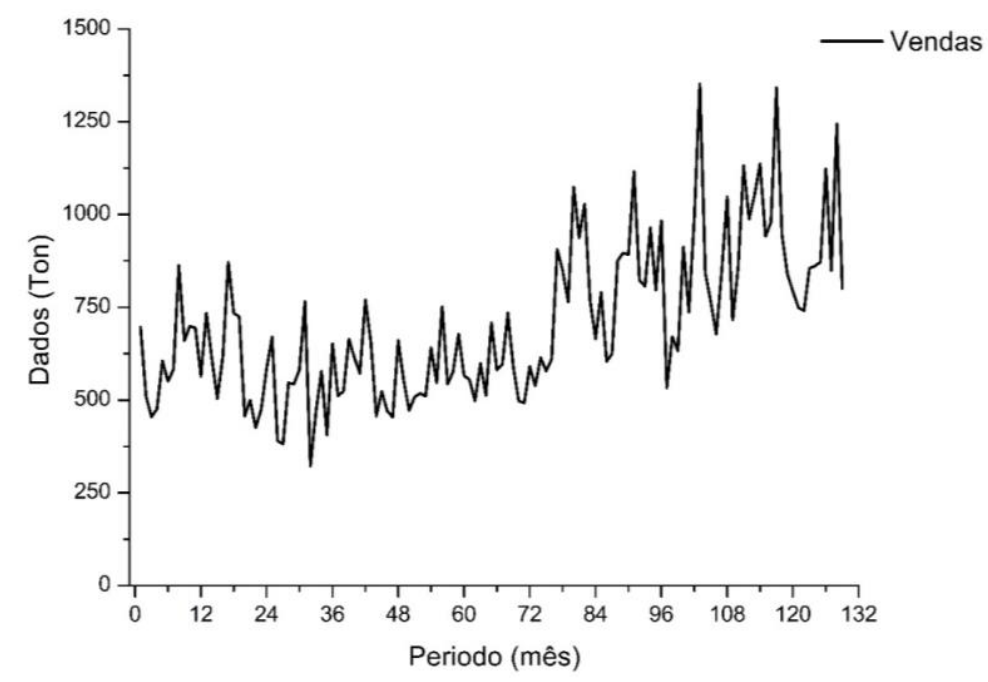

Fonte: Elaborado pelo autor (2019) 
Os testes de Wald-Wolfwitz, Cox-Stuart e Mann-Kendall mostraram p-valor muito próximo de zero, comprovando que se deve rejeitar a hipótese nula e aceitar a hipótese alternativa de que há tendência. A série apresenta tendência de crescimento como pode ser observado na Figura 2. Isso pode ser explicado pelo investimento da empresa em melhorias no processo de irrigação, gestão do solo e monitoramento de pragas e doenças que são fatores essenciais para 0 desenvolvimento do cultivo. Essa variedade apresentou resultados positivos e 0 mercado se mostrou receptivo ao aumento dessa oferta.

Ao final dessa análise temos que a banana prata apresentou em seu histórico de dados um comportamento sazonal com uma frequência de 12 meses e tendência de crescimento.

Com o auxílio do software Action Stat aplicou-se dois métodos para identificar aquele que melhor se adequasse aos dados das séries. Aplicou-se o método ARIMA e Holt-Winters, realizando uma previsão para 6 meses onde os valores da previsão para os dois modelos são apresentados na Tabela 1.

Tabela 1 - Resultado da previsão para a banana prata (em toneladas)

\begin{tabular}{lllll}
\hline Modelo & Mês & Limite inferior 95\% & Previsão & Limite superior 95\% \\
\hline \multirow{4}{*}{ ARIMA } & Out-2019 & 630,847 & 916,445 & 1202,043 \\
& Nov-2019 & 645,264 & 946,567 & 1247,871 \\
& Dez-2019 & 650,614 & 956,068 & 1261,523 \\
& Jan-2020 & 652,637 & 960,585 & 1268,533 \\
& Fev-2020 & 653,802 & 963,897 & 1273,993 \\
& Mar-2020 & 654,764 & 966,918 & 1279,072 \\
\hline \multirow{4}{*}{ Holt-Winters } & Out-2019 & 786,470 & 878,979 & 971,489 \\
& Nov-2019 & 736,559 & 838,369 & 940,180 \\
& Dez-2019 & 805,857 & 921,106 & 1036,355 \\
& Jan-2020 & 708,108 & 827,358 & 946,607 \\
& Fev-2020 & 664,686 & 790,676 & 916,665 \\
& Mar-2020 & 705,589 & 846,066 & 986,543 \\
\hline
\end{tabular}

Fonte: Elaborado pelo autor (2019)

Os dois modelos resultaram em previsões distintas, porém não discrepante uma da outra, a representação gráfica da previsão e o ajuste dos modelos aos dados são apresentados para o modelo ARIMA e Holt-Winters nas Figuras 3 e 4. 
Figura 2 - Tendência da banana prata

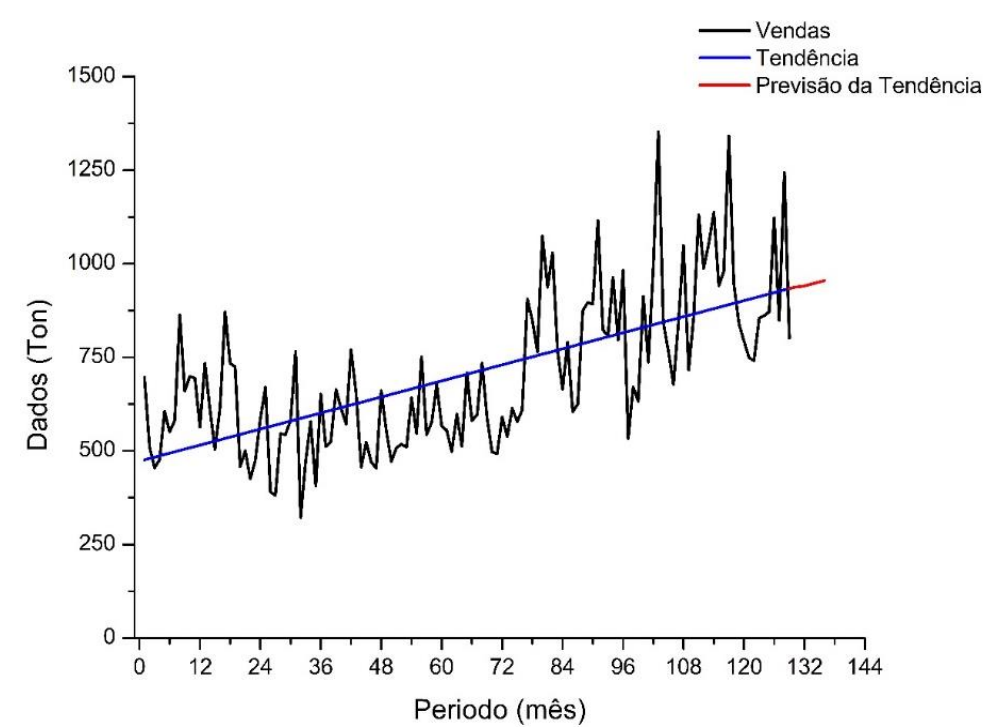

Fonte: Elaborado pelo autor (2019)

O modelo ARIMA teve como resultado um modelo $(1,1,1)$ o que significa que na ordem $p$ temos um processo autorregressivo de primeira ordem que se baseia no período anterior, o último, para gerar o valor futuro. Na ordem d a série necessitou ser diferenciada uma vez para que se tornasse estacionária e para a ordem q, temse uma série com média móvel de primeira ordem, ou seja, em toda a série foi identificado somente uma média dos termos de erro atuais e passados.

Figura 3 - Modelo ARIMA $(1,1,1)$

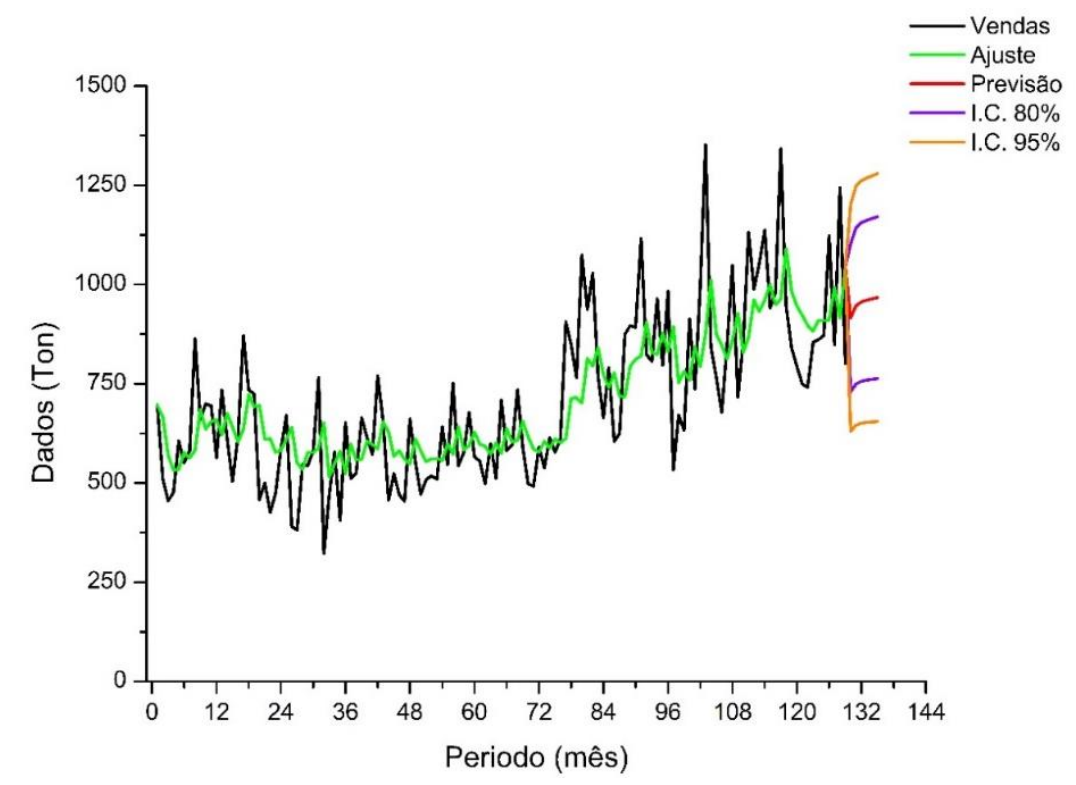

Fonte: Elaborado pelo autor (2019) 
Figura 4 - Modelo Holt-Winters

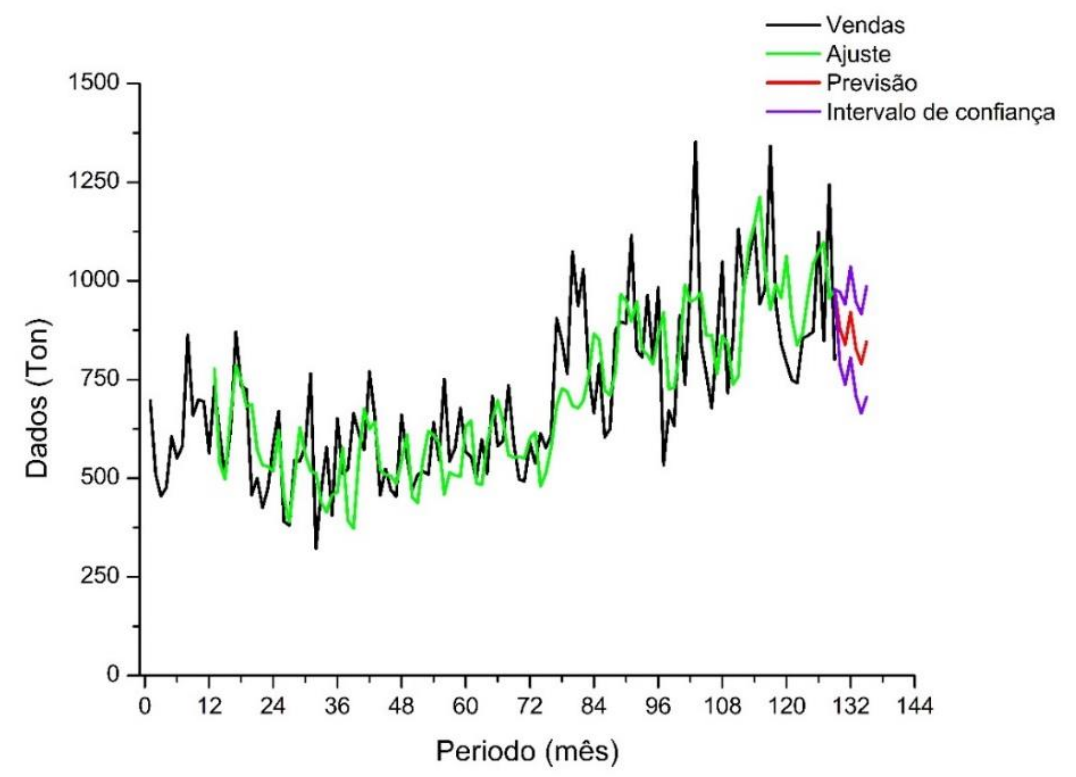

Fonte: Elaborado pelo autor (2019)

No modelo de Holt-Winters obteve-se como constantes de suavização para o nível 0,149 , para a tendência 0,036 e sazonalidade 0,201 , esses valores foram calculados pelo aplicativo.

Para identificar o método com melhor acurácia foi utilizado como medidas de acurácia o MAPE, MAD e MSD. A comparação entre entre os dois métodos de previsão é mostrada na Tabela 2.

Tabela 2 - Medidas de acurácia banana prata

\begin{tabular}{lll}
\hline Medidas de acurácia & ARIMA & Holt-Winters \\
\hline MAPE & 15,79 & 16,29 \\
MAD & 113,10 & 111,70 \\
MSD & 21068,52 & 21706,75
\end{tabular}

Fonte: Elaborado pelo autor (2019)

Observando os resultados da Tabela 2 identifica-se que o menor MAD, ou seja, uma menor dispersão entre os erros foi do modelo Holt-Winters, porém o MAPE e o MSD de menor valor foi do modelo ARIMA que nos mostra que o modelo teve uma previsão que se afastou menos do valores reais e com um erro médio quadrático menor. Assim podemos concluir que o método que apresentou melhor acurácia para os dados foi o ARIMA.

\section{CONSIDERAÇÕES}

Esse trabalho identificou que histórico de vendas da banana prata numa empresa produtora de bananas possui comportamento sazonal e tendência de Revista Produção Online. Florianópolis, SC, v. 20, n. 3, p. 948-967, 2019. 
crescimento. Foram aplicados os métodos de previsão de Holt-Winters e ARIMA. O método que se mostrou mais apropriado de acordo com as medidas de acurácia foi o ARIMA $(1,1,1)$. Os resultados mostraram que a fruta apresenta perspectiva promissora, com uma forte tendência de crescimento. Com esse modelo de previsão espera-se que a empresa possa verificar se a quantidade prevista para as vendas estará disponível no campo, e se necessário já planejar as ações pertinentes com antecipação. Mostrou-se importância de se ter histórico das vendas para realizar projeções futuras que auxiliarão nos processos de decisão e gestão de recursos produtivos. Também foram criadas oportunidades para um aprofundamento na compreensão da demanda de bananas que podem auxiliar no melhoramento dos modelos de previsão ou a união deles.

\section{REFERÊNCIAS}

ACTION, P. Action stat, 2019. Disponível em: http://www.portalaction.com.br/action-statpro.

ALMEIDA, C. O. DE; SOUZA, J. DA S.; CORDEIRO, Z. J. M. Aspectos socioeconômicos. In: CORDEIRO, Z. J. M. (Ed.). . Banana Produção: aspectos técnicos. 1. ed. Brasília: Embrapa Comunicação para Transferência de Tecnologia, 2000. p. 10-11.

BOX, G. E. P. et al. Time series analysis forecasting and control. 4 ed. New Jersey e Canadá: Wiley, 2016.

CHASE, R. B.; JACOBS, F. R.; AQUILANO, N. J. Administração da produção e operações para vantagens competitivas. 11. ed. São Paulo-SP: AMGH, 2006.

CHOPRA, S.; MEINDL, P. Gerenciamento da cadeia de suprimentos: estratégia, planejamento e operação. 1. ed. São Paulo: Pearson Prentice Hall, 2003.

CORRÊA, H. L.; GIANESI, I. G. N.; CAON, M. Planejamento, programação e controle da produção: MRP II/ERP: conceitos, uso e implantação. 4. ed. São Paulo: Editora Atlas, 2006.

CORRÊA, H. L.; GIANESI, I. G. N.; CAON, M. Planejamento, programação e controle da produção: MRP II/ ERP: conceitos, uso e implantação: base para SAP, Oracle Applications e outros software integrados de gestão. 5. ed. São Paulo: Atlas, 2007.

FERNANDES, F. C. F.; GODINHO FILHO, M. Planejamento e controle da produção: dos fundamentos ao essencial. 1 ed. 5 re ed. São Paulo: Editora Atlas, 2016.

FIORAVANÇO, J. C. Mercado mundial da banana: produção, comércio e participação brasileira. Informações Econômica, v. 33, n. 10, p. 15-27, 2003.

GONÇALVES, P. C. Logística e cadeia de suprimentos: o essencial. Barueri-SP: Manole, 2013.

GUAJARATI, D. N.; PORTER, D. C. Econometria básica. 5. ed. São Paulo: MC Graw 
Hill/Bookman, 2011.

KIRCHNER, R. Modelagem e previsão por meio de metodologia Box \& Jenkins: Uma ferramenta de gestão. [s.I.] Dissertação (Pós-Graduação em Engenharia de Produção) Universidade Santa Maria, 2006.

KRAJEWSKI, L.; RITZMAN, L.; MALHOTRA, M. Administração de produção e operações. 8. ed. São Paulo: Pearson, 2009.

LÉLIS, E. C. Administração da produção. 1. ed. São Paulo: Pearson Education do Brasil, 2012.

MAKRIDAKIS, S.; WHEELWRIGHT, S. C.; HYNDMAN, R. J. Forecasting: methods and applications. 3rd ed. New York: [s.n.].

MARTINS, P. G.; LAUGENI, F. P. Administração da produção. 3. ed. São Paulo: Saraiva, 2015.

MORETTIN, P. A.; TOLOI, C. M. DE C. Modelos para previsão de séries temporais. 1. ed. Rio de Janeiro: Blucher, 1981.

MORETTIN, P. A.; TOLOI, C. M. DE C. Análise de séries temporais. 2. ed. São Paulo: Egard Blucher, 2006.

PAPALARDO, F.; MACHADO, S. T.; SACOMANO, J. B. Planejamento e controle de produção (PCP) aplicado ao agronegócio. In: REIS, J. G. M. DOS; NETO, P. L. DE O. C. (Eds.). . Engenharia de produção aplicada ao agronegócio. 1. ed. São Paulo: Blucher, 2018. p. $125-148$.

PELLEGRINI, F. R. Metodologia para implementação de sistemas de previsão de demanda. [s.I.] Universidade Federal do Rio Grande do Sul, 2000.

PELLEGRINI, F. R.; FOGLIATTO, F. S. Passos para implantação de sistemas de previsão de demanda: técnicas e estudo de caso. Produção, v. 11, n. 1, p. 43-64, 2001.

https://doi.org/10.1590/S0103-65132001000100004

ROSSI, J. W.; NEVES, CESAR DAS. Econometria e séries temporais: com aplicação a dados da economia brasileira. Rio de Janeiro: Grupo Editorial Nacional, 2014.

SHUMWAY, R. H.; STOFFER, D. S. Time Series Analysis and Its Applications: With R Examples. 3. ed. Nova York, NY, USA: Springer, 2011. https://doi.org/10.1007/978-1-4419$\underline{7865-3}$

SILVA, S. DE O. E et al. Cultivares. In: FERREIRA, C. F. et al. (Eds.). . O agronegócio da banana. 1. ed. Brasília: Embrapa, 2016. p. 137-170.

SLACK, N.; CHAMBERS, S.; JOHNSTON, R. Administração da produção. 4. ed. São Paulo: Editora Atlas, 2015.

SOUZA, L. DA S.; BORGES, A. L.; SILVA, J. T. A. DA; Solo: manejo e conservação. In: FERREIRA, C. F. et al. (Eds.). . Agronegócio da banana. 1. ed. Brasília: Embrapa, 2016. p. 277-330.

TUBINO, D. F. Planejamento e controle da produção: teoria e prática. 2. ed. São Paulo: Editora Atlas, 2009. 
TUBINO, D. F. Planejamento e controle da produção: teoria e prática. São Paulo: Atlas, 2017.

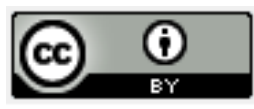

Artigo recebido em: 05/04/2020 e aceito para publicação em: 06/09/2020

DOI: $\underline{10.14488 / 1676-1901 . v 20 i 3.4012}$ 


\section{ANEXOS}

Anexo 1 - Série histórica banana prata (em tonelada)

\begin{tabular}{cccccccccccc}
\hline Mês/ano & $\mathbf{2 0 0 9}$ & $\mathbf{2 0 1 0}$ & $\mathbf{2 0 1 1}$ & $\mathbf{2 0 1 2}$ & $\mathbf{2 0 1 3}$ & $\mathbf{2 0 1 4}$ & $\mathbf{2 0 1 5}$ & $\mathbf{2 0 1 6}$ & $\mathbf{2 0 1 7}$ & $\mathbf{2 0 1 8}$ & $\mathbf{2 0 1 9}$ \\
\hline Jan & 696,46 & 734,06 & 669,80 & 511,50 & 552,38 & 554,44 & 538,26 & 790,14 & 532,88 & 715,92 & 748,86 \\
Fev & 510,12 & 611,22 & 390,29 & 523,71 & 472,01 & 498,30 & 613,77 & 604,54 & 670,96 & 856,77 & 741,25 \\
Mar & 454,91 & 503,69 & 381,32 & 664,10 & 508,45 & 597,83 & 577,82 & 624,62 & 632,79 & 1131,26 & 854,78 \\
Abr & 476,07 & 617,65 & 546,13 & 613,86 & 517,63 & 512,06 & 609,92 & 874,64 & 912,25 & 987,90 & 861,17 \\
Mai & 605,63 & 871,29 & 543,37 & 571,86 & 510,07 & 708,41 & 906,02 & 896,51 & 737,12 & 1052,76 & 871,77 \\
Jun & 550,74 & 734,18 & 581,97 & 770,19 & 641,29 & 581,20 & 847,74 & 891,89 & 994,02 & 1137,16 & 1122,81 \\
Jul & 581,50 & 724,76 & 765,57 & 656,48 & 546,35 & 596,03 & 765,13 & 1115,92 & 1352,07 & 940,95 & 848,51 \\
Ago & 863,16 & 457,36 & 322,07 & 456,83 & 751,41 & 734,55 & 1074,24 & 823,35 & 843,18 & 979,53 & 1244,02 \\
Set & 659,70 & 500,07 & 470,31 & 523,33 & 543,42 & 588,76 & 936,91 & 806,66 & 764,33 & 1341,51 & 801,01 \\
Out & 699,07 & 425,33 & 578,14 & 470,24 & 576,45 & 497,38 & 1028,66 & 964,21 & 677,89 & 945,87 & - \\
Nov & 694,12 & 473,86 & 405,70 & 453,82 & 677,80 & 492,15 & 767,75 & 796,22 & 846,41 & 839,57 & - \\
Dez & 563,73 & 584,49 & 651,80 & 660,69 & 566,41 & 590,66 & 664,73 & 983,19 & 1048,28 & 792,68 & - \\
\hline
\end{tabular}

Fonte: Elaborado pelo autor (2019) 